upregulation of TGF- $\beta$ signalling. Bintrafusp alfa is a first-inclass bifunctional fusion protein composed of the extracellular domain of the TGF- $\beta$ RII receptor (a TGF- $\beta$ 'trap') fused to a human immunoglobulin G1 monoclonal antibody blocking PDL1. We report pooled safety and efficacy in patients with pretreated, immune checkpoint inhibitor-naive, recurrent/metastatic cervical cancer treated with bintrafusp alfa in phase 1 (INTR@PID 001; NCT02517398) and phase 2 (study 012; NCT03427411) studies.

Methodology Patients received bintrafusp alfa $0.3-30 \mathrm{mg} / \mathrm{kg}$ (phase 1 dose escalation) or $1200 \mathrm{mg}$ every 2 weeks (phase 1 dose expansion and phase 2) until progressive disease, unacceptable toxicity, or withdrawal. Treatment past progression was allowed. Primary endpoints were safety (phase 1 dose escalation) and best overall response per RECIST 1.1 (phase 1 dose expansion and phase 2).

Result(s)* As of May 15, 2020 (phase 1) and December 22, 2020 (phase 2), 39 patients had received bintrafusp alfa for a median duration of 2.8 months (range, 0.5-19.3). The median follow-up to data cutoff was 35.0 months and 24.1 months for the phase 1 and phase 2 studies, respectively. All patients had received prior anticancer therapy; 16 (41.0\%) had received $\geq 3$ regimens. Confirmed ORR was $28.2 \%$ (table 1 ); responses occurred irrespective of Moore criteria (phase 1), tumour histology, prior bevacizumab treatment, or radiation treatment. Median overall survival was 13.4 months. No new safety signals and no treatment-related deaths were observed; side effects were manageable.

Conclusion* Bintrafusp alfa had a manageable safety profile and demonstrated clinical activity in patients with heavily pretreated, immune checkpoint inhibitor-naive recurrent/metastatic cervical cancer.

(C) 2021 American Society of Clinical Oncology, Inc. Reused with permission. This abstract was accepted and previously presented at the 2021 ASCO Annual Meeting. All rights reserved.

\section{SINGLE INSTITUTION OUTCOMES FOR CERVICAL CANCER PATIENTS TREATED RADICALLY WITH EBRT USING A PHASE 2 TECHNIQUE WHEN HDR BRACHYTHERAPY WAS NOT POSSIBLE}

J Morgan*, M Elahi, M Zahra, A Stillie. Western General Hospital Edinburgh Cancer Centre, Oncology Department, Edinburgh, UK

\subsection{6/ijgc-2021-ESGO.9}

Introduction/Background* To review overall survival (OS) and disease-free survival (DFS) for cervical cancer patients, unable to receive high dose rate brachytherapy (HDR BT), treated with external beam radiotherapy (EBRT) phase 2 boost

Methodology Data was retrospectively reviewed for patients treated with EBRT phase 2 boost for cervical cancer between 2011 and 2018. Staging, outcomes, recurrence rate and rationale for omitting HDR BT was recorded. The EBRT dose was $45 \mathrm{~Gy}$ in 25 fractions to the pelvis +/- para-aortic area, followed by a phase 2 treatment of $20 \mathrm{~Gy}$ in 10 fractions to residual primary disease. OS and DFS were calculated as a whole and for subgroups based on stage. The Kaplan-Meier method was used with log rank value to assess statistical significance, with two-tailed significance testing and with a p- value $<0.05$ deemed significant. All analysis was done using IBM SPSS statistical software version 24 .

Result(s)* A total of 110 patients received a phase 2 boost over this 8 year period. The median age was 61 years (min 18 to $\max 87$ ). The median radiotherapy dose was 64Gy (min 14Gy- max65Gy). Reasons for phase 2 include co morbidity (42\%), technical limitations (30\%), patient choice $(11 \%)$ and poor compliance (17\%).

The stage (FIGO 2009) distribution was: stage $1(\mathrm{~N}=3)$, stage II $(\mathrm{N}=56)$, stage III $(\mathrm{N}=28)$ and stage IVA $(\mathrm{N}=23)$. The five-year DFS and OS for the entire cohort was $46 \%$ and $27 \%$ respectively. Analysing by stage the 5 year DFS for stage I was $33 \%$, stage II was $46 \%$, stage III was $50 \%$ and stage IVA was $43 \%$. The 5 year OS for stage I was $33 \%$, stage II was $34 \%$, stage III was $25 \%$ and stage IVA was $13 \%$. 5 -year OS for reasons precluding HDR BT were technical limitations (24\%), Co-morbidity (28\%), patient choice $(50 \%)$ and poor compliance $(16 \%)$.

\section{Abstract 117 Table 1}

\begin{tabular}{|l|l|l|}
\hline Number & Reason for EBRT & 5 -year OS \\
\hline 1 & Tumour not amenable & $24 \%$ \\
\hline 2 & Co-morbidities/anaesthetic risk & $28 \%$ \\
\hline 3 & Patient Choice & $50 \%$ \\
\hline 4 & Other (Poor compliance) & $16 \%$ \\
\hline
\end{tabular}

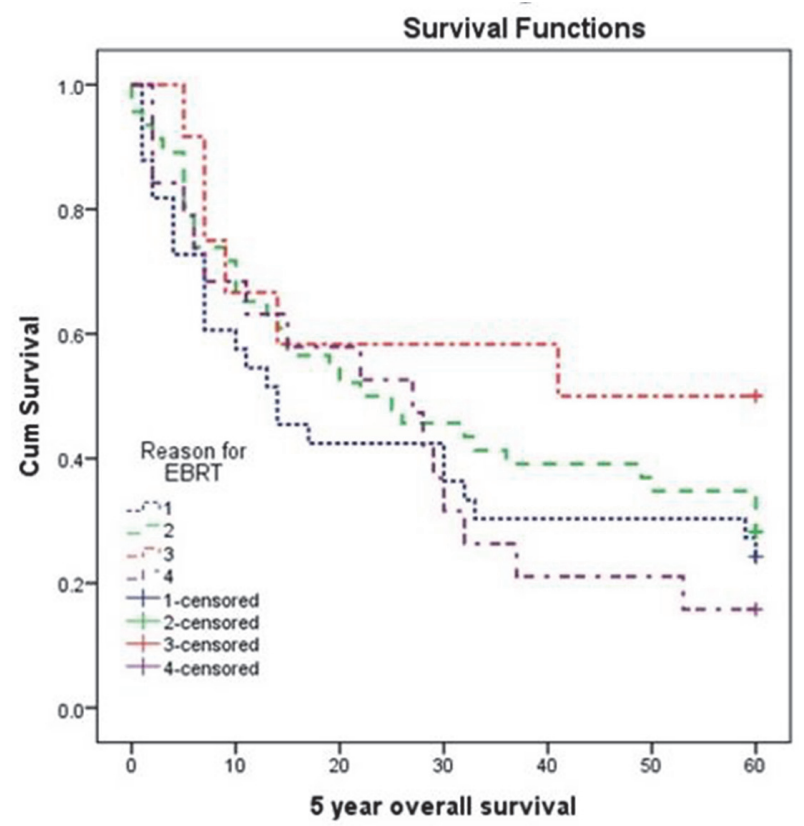

Abstract 117 Figure 1

Conclusion* This data demonstrates that OS and DFS for patients receiving EBRT phase 2 boost are inferior to those who receive $\mathrm{HDR} \mathrm{BT}$, irrespective of stage. It highlights the integral role of HDR BT in the treatment of cervical cancer and patients should be aware that omission can compromise outcome. 